\title{
Editorial
}

\section{General considerations for integrating pharmacogenomics into mainstream medical practice}

Pharmacogenomics is referred to as the scientific field that studies the influence of genetic variation on drug response by correlating gene expression or single nucleotide polymorphisms (SNPs) with a drug's efficacy or toxicity. Pharmacogenomics aims to develop the rationale for individualising drug therapy, based on a patient's genetic profile, to maximise drug efficacy and/or minimise adverse drug reactions (ADRs). Such innovative approaches, where drugs or drugs and food combinations are optimised against an individual's unique genetic profile, would constitute 'personalised medicine'. This notion stems from ancient times, when Hippocrates first said that '.. it is more important to know which kind of person suffers from a certain disease than knowing from which disease somebody suffers'. The recent technological revolution that led to the various high-throughput methods for determining an individual's genetic make-up - namely, next-generation sequencing and microarray-based technologies - has brought the prospect of personalised medicine even closer to fruition, but, at the same time, has posed some dilemmas and has serious ethical implications that have to be overcome and addressed properly.

The first important issue is the choice of the genotyping platform, which is directly dependent on the cost of the pharmacogenomic test that is being offered. Despite the fact that there are numerous mutation detection and screening methods, there is no single platform or methodology that prevails for pharmacogenetic testing.
Genotyping can be done using different approaches with respect to the throughput, amplification-based and separation technologies and labelling. The variety of detection approaches makes it not only difficult, but also challenging to determine which is better suited to a laboratory setting, although DNA sequencing is considered to be the gold standard for the identification of DNA sequence variants, particularly with the advent of the next-generation sequencing technologies. The initial investment costs and the expected test volumes are some of the factors that need to be taken into consideration prior to choosing the genotyping technique, including hardware and software, testing reagents, and kits. The choice of genotyping platform goes hand in hand with the training of the personnel - not only of those performing, but also those interpreting the pharmacogenomic test. The continuing education of personnel in the diagnostic laboratory is crucial for the accuracy of the results obtained (see also below).

Alongside this, and closely related to it, healthcare costs represent an important parameter. Generally, the cheaper the pharmacogenomic test, the more likely it is to be undertaken by a patient or prescribed by the physician. Unfortunately, economic barriers to the adoption of genomic data for individualising medical practice exist and mostly depend on the nature of the technology used. Different reimbursement policies from different insurance companies can also pose an obstacle to the rapid dissemination of pharmacogenomics. In 
other words, it is only when insurance companies start paying for pharmacogenomic tests to individualise drug prescription, or to prevent or effectively manage chronic diseases, that personalised medicine will start to flourish. Using the example of warfarin, however, shows that this perspective is far from becoming a reality. Although information was added to the warfarin label by the US Food and Drug Administration in 2007, based on the influence of the CYP2C9 and VKORC1 genes on anticoagulation-related treatment, reimbursement of this genetic test for warfarin dosing is not uniform among beneficiaries. Also, with respect to the impact of pharmacogenomics on reducing overall healthcare costs, several reports have reported cost-effectiveness analyses on drug treatments for cancer (eg HER2 pharmacogenetic testing), psychiatry and chronic inflammatory diseases, and, hence, demonstrated the cost benefits of a putative pharmacogenetic test. It is clear that further debate is required among healthcare decision makers to be able to realise the expected economic benefits, both for pharmaceutical companies and public health. Interested parties may also influence the adoption of pharmacogenetic tests, since they can choose to reimburse the costs if they are convinced that these tests can have a positive impact on their drug treatment.

Another important parameter that will facilitate incorporation of pharmacogenomics into mainstream medical practice is the education of healthcare professionals. So far, the introduction of pharmacogenomic testing in the clinic has not met with the reciprocal and continuing education of physicians, particularly senior ones, which is a cumbersome issue, particularly in light of applying these tools to a growing number of patients. ${ }^{1}$ This not only applies to basic pharmacogenomic knowledge, but also to the interpretation of pharmacogenomic test results and their utilisation in the clinical setting for the therapeutic management of patients. In fact, this deficiency is part of a general lack of preparation by the primary healthcare workforce to facilitate the integration of genome medicine into mainstream clinical practice. In this respect, it is imperative that biomedical curricula in higher education institutions are enriched with relevant courses on pharmacogenomics and personalised medicine. Indeed, the International Society of Pharmacogenomics Education Forum has already requested such measures. Clearly, patients expect to receive pharmacogenetic services from healthcare professionals who can confidently and convincingly explain the test and prescribe the proper drug, at the correct dose, based on the pharmacogenomic test result. The appropriate education of healthcare professionals is vital to reach this point. Furthermore, the basics of pharmacogenomics should also be disseminated to a much wider audience - namely, the general public — especially to explain to them why such tests should only be ordered through a medical practitioner and alert them to the fundamental differences between pharmacogenomics and 'predictive genomics', the latter currently standing on weak scientific foundations. Preliminary data from a survey undertaken by our group, using a very large sample, show that the general public is largely against direct-to-consumer genetic testing, including pharmacogenomic testing (paper in preparation).

Most importantly, the complete implementation of pharmacogenomics and personalised medicine will only take place when they are properly regulated by healthcare authorities. By definition, pharmacogenomics investigates inter-individual differences in genetic make-up; therefore, issues pertaining to genetic discrimination - and privacy - particularly in relation to the accessing of people's genetic composition by life and healthcare insurance companies, should be resolved. The social consequences arising from new terms such as 'responder' or 'nonresponder' to a given drug treatment could involve genetic stigmatisation or personal identification issues. Similarly, pharmaceutical companies, for economic reasons, may simply ignore patients who are either suffering from rare or complex genetic disorders or who are not expected to respond to any known treatment, leading to denial of effective treatments. Overall, genomic data are very different from traditional medical information, the latter being self-limited and often transient, particularly when data storage comes into play. ${ }^{2}$ In this case, 
serious concerns are raised about the potential for loss of confidentiality or privacy that should be safeguarded appropriately. In an attempt to regulate these questions by means of legislation, national authorities and governments have enacted specific measures, such as the Genetic Information Non-discrimination Act (GINA; http://www. genome.gov/24519851) and the Health Insurance Portability and Accountability Act Privacy Rule (http://www.hhs.gov/ocr/privacy/hipaa/administra tive/privacyrule/index.html) in the USA.

One way to resolve this deficiency is meticulously to collect and document patients' genomic data in central data depositories (eg clinical genetics databases) that fulfil strict data protection criteria and carefully control data access. Such databases would be located in various healthcare institutions and record phenotype, genotype and personal data but access to these data would be restricted by user rights. User identification would be performed based on a number of methods, such as use of passwords, access cards or other means of unique

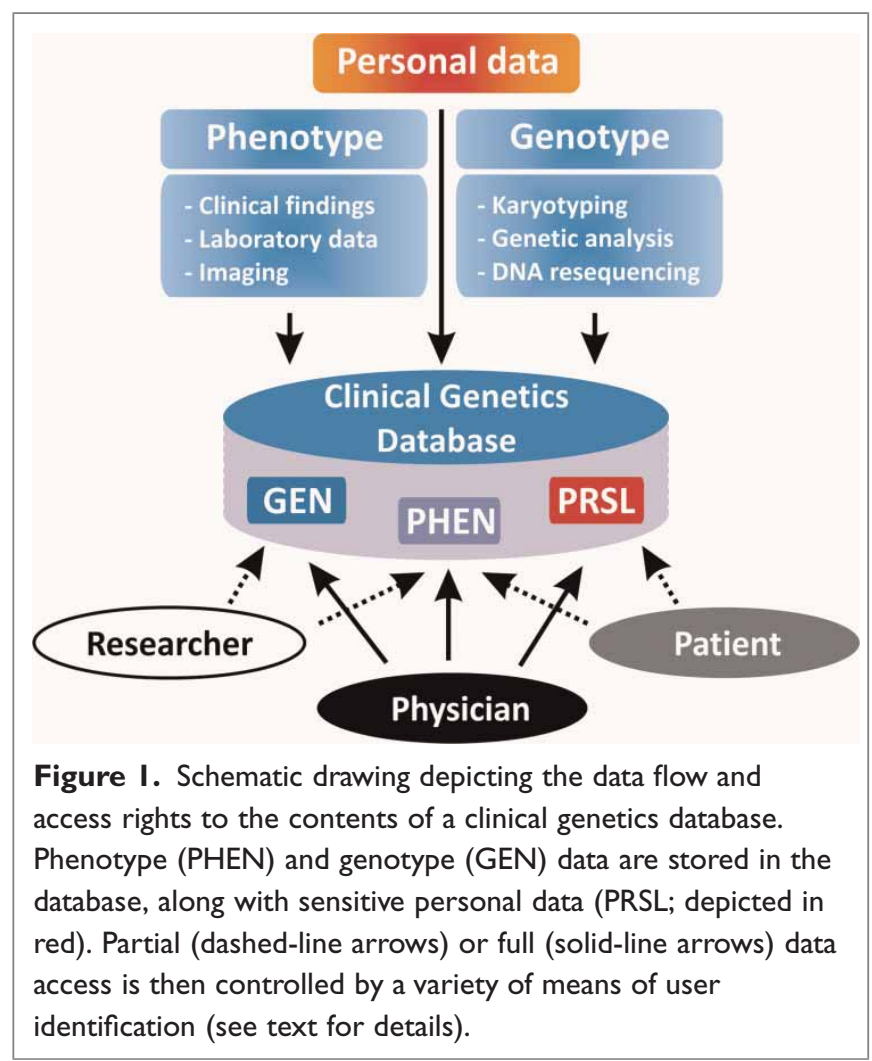

researcher identification (eg ResearcherIDs (http:// www.researcherid.com) and/or Researcher Identification Primers (RIPs; http://www.gen2phen. org) and/or a double-layered authentication mechanism, determined by the user's password and IP address). In this way, researchers and patients would be granted only partial access to their stored data and only the physician would be granted full access to an individual's clinical picture and genetic background (Figure 1). Certainly, when whole-genome sequencing becomes routine, and personalised medicine is common, these databases may well be something we take for granted. In the meantime, the first efforts in that direction have already started, with prototype software from our group that allows an individual's genetic profile to be stored (with informed consent) so that this information can be retrieved only by the patient and his/her physician (unpublished results). A convenient graphical interface is provided to the end user, who can enter data and retrieve results (through queries) from the clinical genetics database in which the relevant information has been stored. ${ }^{3}$ Such a database, currently under development, would allow patients securely to store all their genetic and related phenotypic information, hence contributing decisively to customised medical treatment.

Of course, the development of such repositories raises particularly complex ethical challenges. Primarily, the inclusion of clinical and molecular data connected to specific individuals must be done in a way that ensures anonymity. How best to achieve this has not yet been established, but it is widely agreed that strict governance frameworks must be established to address any and all confidentiality concerns. This is a particularly timely issue, following the recent work of Homer and co-workers, ${ }^{4}$ which created much debate - and some frustration - in the human genomics community about how we can share summary-level genetic association data optimally and safely. These authors showed that summary-level genotypic data do not completely mask a person's identity, so that greater emphasis and caution are needed for the confidential sharing and combining of genomics 
data from patients and non-affected individuals in order fully to mask personal identity.

In essence, the effective utilisation of pharmacogenomics relies heavily on a variety of parameters that are currently being addressed to a greater or lesser extent. We stand at the dawn of personalised medicine, and the success of this endeavour will depend on both deciphering and gaining a deeper understanding of the intricacies that regulate and underlie an individual's 'genoprint', particularly variability in response to medication.

George P. Patrinos

Department of Pharmacy School of Health Sciences

University of Patras, Greece Tel/Fax: +302610969834;

E-mail:gpatrinos@upatras.gr

\section{Acknowledgments}

I am grateful to Dr Marianthi Georgitsi for critical reading of the manuscript and for her detailed and constructive comments. Part of our work has been supported by European Commission grants [MEDGENET (FP6-31968), EuroGenTest (FP6-512148) and GEN2PHEN (FP7-200754)] and the University of Patras research budget.

\section{References}

1. Frueh, FW. and Gurwitz, D. (2004), 'From pharmacogenetics to personalized medicine: A vital need for educating health professionals and the community', Pharmacogenomics Vol. 5, pp. 571-579.

2. Povey, S., Al Aqeel, A.I., Cambon-Thomsen, A., Dalgleish, R. et al. (2010), 'Practical guidelines addressing ethical issues pertaining to the curation of human locus-specific variation databases (LSDBs)', Hum. Mutat., In press.

3. van Baal, S., Zlotogora, J., Lagoumintzis, G., Gkantouna, V. et al. (2010), 'ETHNOS: A versatile electronic tool for the development and curation of national genetic databases', Hum. Genomics, Vol. 4, No. 5, pp. 361-368.

4. Homer, N., Szelinger, S., Redman, M., Duggan, D. et al. (2008), 'Resolving individuals contributing trace amounts of DNA to highly complex mixtures using high-density SNP genotyping microarrays', PLoS Genet. Vol. 4, p. e1000167. 